\title{
Algorithm analyzer to check the efficiency of codes
}

\begin{abstract}
Efficiency of codes developed is always an issue in software development. Software can be said to be of good quality if the measurable features of the software can be quantitatively checked for adoption of standards or following certain set rules. Software metrics can therefore come into play in the sense of helping to measure certain characteristics of software. The issue and factors pertaining to efficiency of a code will be addressed by software metrics. Existing tools that are used to analyze several software metrics have come a long way in helping to assess this very important part of software development. This paper described how software metrics can be used in analyzing efficiency of the developed code in early stage of development. A tool (algorithm analyzer) was developed to enable analyze a given code to check its efficiency level and produce efficiency reports based on the analysis. The system is able to help the code checking whilst maintaining the standards of coding for its users. With the reports that are generated it would be easy for users to determine the efficiency of their object oriented codes.
\end{abstract}

Keyword: Software metrics; Software measurement; Efficiency 